Commun. math. Phys. 21, 314-333 (1971)

(C) by Springer-Verlag 1971

\title{
Difficulty with a Kinematic Concept of Unstable Particles: the SZ.-Nagy Extension and the Matthews-Salam-Zwanziger Representation
}

\author{
DAVID N. WILlIAMS \\ The University of Michigan, Ann Arbor, Mich., USA
}

Received November 15, 1970

\begin{abstract}
We discuss the possibility of describing unstable systems, or dissipative systems in general, by vectors in a Hilbert space, evolving in time according to some nonunitary group or semigroup of translations. If the states of the unstable or dissipative system are embedded in a larger Hilbert space containing "decay products" as well, so that the time evolution of the system as a whole becomes unitary, we show that the infinitesimal generator necessarily has all energies from minus to plus infinity in its spectrum. This result supplements and extends the well-known fact that a positive energy spectrum is incompatible with a decay law bounded by a decreasing exponential. As an example of both facts, we discuss Zwanziger's irreducible, nonunitary representation of the Poincaré group; and we find its minimal, unitary extension (the Sz.-Nagy construction). The answer provides a mathematically canonical approach to the Matthews-Salam theory of wave functions for unstable, elementary particles, where the spectrum difficulty was already recognized. We speculate on the possibility that the Matthews-Salam-Zwanziger representation might be a strong coupling approximation in the relativistic version of the WignerWeisskopf theory, but we have not shown the existence of a physically acceptable model where that is so.
\end{abstract}

\section{Discussion}

There have been some conjectures in recent years that strong interaction resonances in relativistic quantum physics may have a kinematic characterization as "unstable particles," belonging to complex rest mass eigenvalues of a nonunitary representation of the Poincaré group [1-4]. That such resonances might have an intrinsic "integrity" ${ }^{[5]}$ is an appealing idea, suggested by the concept of "nuclear democracy" [6], more particularly by the analytic $S$-matrix philosophy that resonance

${ }^{1}$ Our use of the word "integrity" is less precise than that of Lurçat [5]. We have in mind the same thing that we mean by the equally fuzzy "kinematic concept of unstable particles." We use it as a term whose potential meaning is to be realized by the success of some mathematical scheme such as that discussed here. Vaguely, it should mean that there are characteristic properties of unstable particles that can be isolated independently of any interaction and discussed without the aid of interaction dependent quantities like the $S$ matrix, or the relation between free and total Hamiltonians. 
and stable particle poles are to be treated on the same footing, although they lie on different sheets, and by the Regge phenomenology, which classifies stable and unstable particles alike by Regge trajectories. Indeed, practitioners of the latter philosophy sometimes assign some sort of integrity to entire Regge trajectories, which are to be exchanged just like particles, and which in some theories are to generate "Landau singularities" via iteration of unitarity, just as elementary particles do.

Although analytic $S$-matrix theorists would not feel compelled to do so, if only because Hilbert space has a vestigial role in their concept, these ideas naturally suggest that one should try to associate unstable particles with representations of the Poincare group, by analogy with Wigner's classification of stable, elementary systems according to their masses and spins. Some ways of doing that have been proposed $[1-4]^{2}$.

Although it does not explicitly push the correspondence between elementary systems and representations of the Poincaré group, the work of Matthews and Salam [1] epitomizes both the attractive features and the difficulties in physical interpretation of such an approach. They try to make sense out of the description of an elementary unstable particle by an ensemble of states decaying in proper time in the center of momentum frame by $\exp (-\operatorname{im} \tau-\gamma \tau)$, for $\tau \geqq 0$, where $m>0$ and $\gamma>0$ represent the mass and one-half the width. By Fourier transformation, they get a mass distribution density matrix that is the usual Lorentzian shape, but which includes all masses from plus to minus infinity.

They attempt to associate a local field with the unstable particle, whose Umezawa-Källén-Lehmann spectral function is of the Lorentzian shape, cut off sharply below the physical threshold for the stable decay products. In their model, the field for an unstable elementary particle is conceived as a function of the free fields of the decay products.

Unfortunately, the modern development of rigorous field theory makes it clear that their model is unrealizable, if taken too literally, in which case the unstable particle field would have to be in the Borchers class of the free fields; and up to now no realization exists. Their remarks on the sense in which an elementary unstable particle can be described by a mass wave function in the Hilbert space of free decay products still have a point; and we aim to discuss that below; but without the field theoretical underpinning, their own criterion by which a theory of elementary unstable particles is to be considered successful is not satis-

${ }^{2}$ We do not attempt to review all of the fairly substantial literature on unstable particles. Much of it can be traced from the references we cite. The paper of Lurçat [5] and the book of Newton [7] are especially useful for that purpose. Lest there be some misunderstanding, let us emphasize that it is not our purpose to criticize the orthodox ways of understanding resonances and unstable particles; but rather it is to explore how far a group theoretical description might reasonably be pushed. 
fied; namely, that it should be clear in what sense the kinematic description is or can be an approximation to dynamics.

We accept that as a sensible criterion; but the best we can do in this paper is to formalize some of the reasons why it is difficult to satisfy, and has not been satisfied even up to now, if one insists too strongly on a kinematic concept. We do propose a speculation on how the MatthewsSalam approach might conceivably be justified as a strong-coupling version of the Wigner-Weisskopf model.

Zwanziger's work [2], although done independently, is related to the wave-function aspect of the Matthews-Salam theory. He considers a nonunitary, irreducible representation of the Poincaré group, defined on the space of four-velocity wave functions

$$
\begin{aligned}
& \varphi \in \mathscr{H}_{U}=L_{2}\left(d^{3} u / 2 \sqrt{1+\boldsymbol{u}^{2}}\right) \otimes \mathbb{C}^{2 S+1}, \\
& \langle\varphi, \varphi\rangle=\sum_{\lambda=-s}^{s} \int \frac{d^{3} u}{2 \sqrt{1+\boldsymbol{u}^{2}}} \overline{\varphi(\boldsymbol{u})_{\lambda}} \varphi(\boldsymbol{u})_{\lambda},
\end{aligned}
$$

with four-momentum operators

$$
u_{0}=\sqrt{1+u^{2}}, \quad P_{\mu}=(m-i \gamma) u_{\mu}, \quad m>0, \quad \gamma>0,
$$

which are normal ${ }^{3}$, but not self-adjoint. The translations are represented by the nonunitary operators $T(b)=\exp (i P \cdot b)$, while the Lorentz transformations have the usual, unitary representation for half-integer spin $S$. The squared mass operator is a complex, Poincaré invariant, $P \cdot P$ $=(m-i \gamma)^{2}$.

Zwanziger showed that the vectors in $\mathscr{H}_{U}$ evolve and decay exponentially for positive times ${ }^{4}$

$$
\varphi^{t}=V(t) \varphi \equiv \exp \left(-i P_{0} t\right)
$$

in the way characteristic of an unstable particle of rest mass $m$ and invariant lifetime $\tau=1 / 2 \gamma$. His representation is thus a candidate to realize in an economical way the concept of an unstable particle as an elementary system, transforming according to an irreducible, nonunitary representation of the Poincaré group. This representation was also studied by Kawai and Gotō [4], who discussed its second quantization in a way different from that of Matthews and Salam.

Beltrametti and Luzzato [3] considered a more general class of nonunitary, irreducible representations, of which Zwanziger's becomes a special case.

\footnotetext{
${ }^{3}$ A normal operator commutes with its adjoint.

${ }^{4}$ For negative $t$, the evolution operators are unbounded, and $\varphi^{t}$ will be defined only for vectors in a dense domain. For positive $t, V(t)$ is bounded and no domain questions arise; furthermore it is a contraction, i.e., its bound is not bigger than one, so it does not increase the norm of any vector on which it operates.
} 
None of these authors included a criterion of acceptability like that of Matthews and Salam in the scope of their discussions.

One can imagine variations on this approach to allow reducible representations of the group, especially if he wants to include several spins [8], or a Regge trajectory, in one representation having some properties of integrity whose precise formulation is to be discovered.

We want to discuss the physical interpretation of the general type of theory with a group law of time evolution, but first let us mention the rather different proposal of Lurçat [5]. He describes unstable particles by a multiplicity-free representation of the Poincare group on a space of density matrices belonging to characteristic mass distributions such as one observes in the decay products. So far this is just an abstraction of some key elements of the Matthews-Salam theory, as he points out. But in addition he requires that the density matrices correspond to intrinsically impure statistical mixtures, due to the operation of a continuous superselection rule in the mass. He appears to rule out the possibility of discussing the time evolution or decay law of such states, making a sharp distinction between his concept and that of a metastable state, for which such things make sense. The major criticism of his theory is the same as that of the others; a clearer picture of how the kinematic description can be fitted into some dynamical scheme needs to be developed.

Let us return now to the type of theory where unstable particles correspond to a representation of the Poincare group with nonunitary time evolution. It seems natural to interpret the nonunitarity of the time evolution, or the decay of the unstable particle vectors in $\mathscr{H}_{U}$ for positive times, as being due to a loss of probability from $\mathscr{H}_{U}$ into the Hilbert space of states of the decay products, $\mathscr{H}_{D}$. Unfortunately, the most obvious way of formulating this interpretation leads inevitably to the appearance of unbounded energies, both above and below, in the space of decay products.

This phenomenon has been known for some time, in case the decay law for positive time is exponential [9]. We review that situation in the Appendix. If the time evolution in $\mathscr{H}_{U}$ obeys a group law for positive times (or a semigroup law), and if it is represented by nonunitary contractions that are also normal operators, having a normal infinitesimal generator such that the imaginary part is strictly less than a negative number, as in Zwanziger's theory, that can be shown to enforce a decay law bounded by an exponential. Our main technical result in this paper is a precise statement and proof of the fact that the phenomenon of unbounded negative energies in the spectrum occurs whenever the semigroup law is obeyed, whether or not the exponential bound is valid. 
We formalize the above approach as follows. We assume that an elementary unstable particle has enough integrity to deserve a (separable) Hilbert space of states of its own, $\mathscr{H}_{v}$; and we assume that the evolution of these states for positive times is described by a weakly continuous, one-parameter family of contractions $V(t)$, which form an Abelian semigroup:

$$
V(t) V\left(t^{\prime}\right)=V\left(t+t^{\prime}\right) \text { for } t, t^{\prime} \geqq 0, \quad V(0)=I .
$$

We assume that $V(t)$ is not unitary for any $t>0$. We have in mind that $\varphi^{t}=V(t) \varphi$ decays strongly, but we don't need detailed properties of $V(t)$ for our argument. It need not be defined at all for negative $t$. It is a standard theorem that every continuous, contraction semigroup of the above type has as infinitesimal generator a dissipative operator ${ }^{5}$; and our remarks apply not only to unstable particles but to a certain class of dissipative systems in general. It goes without saying that the time evolution typically does not obey a semigroup law in dissipative situations that arise by considering non-isolated subsystems of a larger, isolated system.

To interpret the nonunitarity of $V(t)$ as being due to the fact that we are restricting ourselves to the space of unstable particle states, we say that if we included the space of the decay products as well, and described the evolution of the system "unstable particle" plus "decay products" as a whole, there would be no loss of probability; the time evolution would be unitary.

Our framework is thus the following: there is a larger Hilbert space $\mathscr{H}$ in which $\mathscr{H}_{U}$ is embedded, $\mathscr{H}_{U} \subset \mathscr{H}$, and there is a continuous, unitary representation $U(t)$ of the time evolution group on $\mathscr{H}$ which becomes $V(t)$ when restricted to positive times and to the subspace $\mathscr{H}_{U}$. Namely, for $t \geqq 0$,

$$
V(t)=\operatorname{pr} U(t) \equiv E_{U} U(t) E_{U},
$$

where $E_{U}$ is the projection operator for $\mathscr{H}_{U}$ in $\mathscr{H}$.

We are always free to decompose $\mathscr{H}$ into a direct sum of $\mathscr{H}_{U}$ and the rest, and if $V(t)$ actually has the property of causing the vectors in $\mathscr{H}_{U}$ to decay strongly, it is natural to call the orthogonal complement of $\mathscr{H}_{U}$ in $\mathscr{H}$ the space of decay products $\mathscr{H}_{D}: \mathscr{H}=\mathscr{H}_{U} \oplus \mathscr{H}_{D}$. In any case we can use $\mathscr{H}_{D}$ as a notation for the orthogonal complement.

Now there is a construction due to Sz.-Nagy [10] that gives, for any weakly continuous representation $V(t)$ of a one-parameter, Abelian semigroup by contractions on a separable Hilbert space $\mathscr{H}_{U}$, an extension to a larger space $\mathscr{H} \supset \mathscr{H}_{U}$, and a unitary group representation $U(t)$ which

${ }^{5}$ If $V(t)=\exp (-i A t)$, the infinitesimal generator $A$ is dissipative when it satisfies $\operatorname{Im} A<0$. Of course the exponential has to be defined when $A$ is not normal. 
extends the semigroup $V(t)$ in the way described above. This construction gives the smallest extension possible, up to an isometric isomorphism. Any other unitary extension of $V(t)$ contains Sz.-Nagy's minimal extension in some subspace. Actually, it is only the minimal extension that is directly relevant for the physical interpretation, because it is characterized by the property that $U(t) \mathscr{H}_{U}$ generates the extension space $\mathscr{H}$. That is, the set of vectors of the form $U(t) \varphi$, with $\varphi$ in $\mathscr{H}_{U}$, spans $\mathscr{H}$. Thus, vectors in $\mathscr{H}_{U}$ cannot evolve into vectors with components outside the minimal extension space; and Sz.-Nagy's extension is the only one that has a right to be associated with decay products of states in $\mathscr{H}_{U}$.

In Section II we use the Sz-Nagy construction to show that the spectrum of the infinitesimal generator of any unitary extension $U(t)$ $=\exp (-i E t)$ of a nonunitary, continuous semigroup has all points from minus to plus infinity in its spectrum. We do that by exhibiting nonzero vectors $\psi$ in $\mathscr{H}_{D}$ which are sharply localized in time; that is, there exists a number $B$ such that $\langle\psi, U(t) \psi\rangle=0$ for all $|t|>B$. That the spectrum of $E$ is the whole real line is then an easy consequence of the PaleyWiener theorem.

Thus our scheme seems to be unphysical; as soon as we discuss the system as a whole, we find that the energy is unbounded from below.

Nevertheless, Zwanziger's proposal of a group representation for elementary unstable particles is appealing, and the fact that we find it difficult to interpret does not mean that we have to abandon it as meaningless. Thus, we have thought it worthwhile in Section III to look at the Sz.-Nagy extension of Zwanziger's representation. The Sz.-Nagy theory is not restricted to one-parameter semigroups, but covers a wide class of several-parameter group structures, and other structures as well. For Zwanziger's representation, it is quite straightforward to reduce the extension problem to a one-parameter problem for the proper-time evolution, and to write down the answer explicitly. The result is a mathematically canonical derivation of the Matthews-Salam wave function (the Lorentzian distribution mentioned earlier), which describes the initially undecayed state in the center-of-momentum energy representation. We call this explicit, unitary extension of Zwanziger's nonunitary representation the MSZ representation.

The interpretation suggested by the Sz.-Nagy extension theorem seems to us a step removed, however, from that of Matthews and Salam. They appear to interpret the Breit-Wigner wave function (cut off below the physical threshold) as the mass distribution of the unstable particle considered as a superposition of decay products; i.e., the unstable particle is just a special vector in the Hilbert space of free decay products. A more orthodox view, and we believe a more natural one, would be that of the 
relativistic version of the Wigner-Weisskopf theory [11, 12], where the undecayed, unstable particle state would lie orthogonal to the space of free decay products and would have, neglecting the negative energy difficulty, an approximately Breit-Wigner distribution in total, interacting mass. The asymptotic states of the unstable particle for large positive time would then lie in the orthogonal space of free decay products; and they would have the same distribution in free mass as the undecayed states in total mass, because the wave operator would map the total mass operator into the continuous part of the free mass operator $^{6}$. For the same reason, the decay law of the asymptotic state according to the free mass operator would be the same as that of the initially undecayed state according to the total mass operator, and many of the statements of Matthews and Salam on time evolution would be recovered, if reworded a bit.?

We have already admitted that we cannot say such a scheme works unless we understand how the MSZ representation, with its negative energies and exponential decay law, can be a natural, first approximation to the "true" dynamical situation. This entails a dilution of any kinematic aspect in the MSZ concept of an unstable particle, but that fact of life is well recognized in the literature. In the simple, exactly soluble version of the Wigner-Weisskopf model, [11, 12, 14-17] where the interaction has nonvanishing matrix elements only between the undecayed, discrete energy state and continuum energy states of the free decay products (weak coupling approximation), it is well understood in what sense the exponential decay is a first approximation, arising from the presence of a pole on the second sheet of the appropriate matrix element of the resolvent of the full Hamiltonian. By making the exponential decay approximation to the weak coupling model, one would recover Zwanziger's representation in the subspace $\mathscr{H}_{v}$. The Sz.-Nagy extension of that approximation would then have no obvious relevance to the weakcoupling mass operator from which one started, nor indeed to the original Hilbert space.

That is perhaps the correct attitude, and we lean towards it ourselves, but in Section IV we speculate on another possibility that assigns a basic role to the MSZ representation. We propose a more complicated WignerWeisskopf model, where the interaction part only of the time evolution is given by the Sz.-Nagy extension of Zwanziger's representation. In other words, maybe the MSZ representation can be understood as the

${ }^{6}$ Coester [13] has emphasized that the relativistic theory of wave operators in the center of mass closely parallels the non-relativistic theory, for a physically nontrivial class of models.

7 Unless the above is possibly the interpretation they intend. Their discussion is not very detailed on this point. 
strong-coupling approximation to a relativistic, "two-level" theory, one level being the undecayed, unstable state and the other the continuum of free decay products. The negative energy problem is thereby avoided, because there is no physical reason why the spectrum of the interaction mass should not go to minus infinity.

It is of course not intuitive nonsense to think that the exponential decay law, in the case of strongly decaying elementary particles, might be a strong-coupling approximation; but we want to emphasize that this hypothesis applied to the MSZ theory is highly speculative, and perhaps even impossible. In Section IV we discuss the mathematical formulation of such a theory, and we point out that it is nontrivial to prove the existence of a total mass operator that is selfadjoint and positive. At least we have been unable to do so up to now.

We do remark that if such a total mass operator exists, its kinetic part cannot dominate its interaction part in the Kato sense [18]. In particular, the kinetic part always has vectors in its domain that are not in the domain of the interaction part, so that if the total mass can be defined at all in the Kato sense (which of course is not the only possibility), it has to be the interaction part which dominates the kinetic part. Thus we use the speculative language: if the theory is defined at all, it may be an intrinsically strong coupling theory ${ }^{8}$. From this one can see why the positivity condition might be delicate; the spectrum of the interaction part must still be perturbed strongly enough to remove the negative energies.

In any case, the physical content of the Wigner-Weisskopf model is stable against a wide range of modifications that affect the dynamical details but not those features that make us willing to say it describes an unstable system, and we think it reasonable to expect that a physically meaningful interpretation of Zwanziger's representation and its extension should fit into that framework.

\section{Trouble with a Semigroup Law}

We aim to prove the following theorem:

Theorem. Let $V(t)$ be a weakly continuous, one-parameter semigroup of contractions on a separable Hilbert space $\mathscr{H}_{U}$, satisfying $V(t) V\left(t^{\prime}\right)$ $=V\left(t+t^{\prime}\right)$ for $t, t^{\prime} \geqq 0$, and $V(0)=I$. Let $V(t)$ be nonunitary for all $t>0$. Let $U(t)$ be any extension of $V(t)$ to a weakly continuous, unitary representation of the one-dimensional translation group on a larger Hilbert space $\mathscr{H} \supset \mathscr{H}_{U}$, such that $V(t)=\operatorname{pr} U(t)$ for $t \geqq 0$.

Then the spectrum of the infinitesimal generator $E$ of $U(t)=\exp (-i E t)$ is the whole real line.

\footnotetext{
${ }^{8}$ This mathematical statement is not a priori related to the shortness of the lifetime.
} 
We have already indicated that it is sufficient to prove this theorem for Sz.-Nagy's minimal construction. His construction is minimal in the sense that $\mathscr{H}$ is spanned by vectors of the form $U(t) \varphi$ where $\varphi \in \mathscr{H}_{v}$. He showed that all such minimal extensions are isomorphic and isometric. Since any unitary extension contains an invariant subspace of that form, on which the restriction of $U(t)$ is clearly unitary, the theorem is reduced to the case of Sz.-Nagy's construction.

Now let us review his construction ${ }^{9}$, because we have to use its detailed properties.

First, we define $V(-t)=V(t) *$ for $t>0$.

Consider the vector space of all functions of $s$ with values in $\mathscr{H}_{U}$, where $-\infty<s<\infty$, and where the operations of vector addition and scalar multiplication are defined in the obvious way. In this space, Sz.-Nagy identifies two linear submanifolds, $F$ and $G$. The manifold $G$ consists of those functions $g(s)$ that vanish for all but a finite number of values of $s$. The manifold $F$ consists of those functions of the form

$$
f(s)=\sum_{u} V(u-s) g(u),
$$

where $g \in G$ and the sum is over the values of $u$ for which $g(u) \neq 0$.

It is the manifold $F$ that is going to correspond to the extension space $\mathscr{H}$. We make $F$ into a pre-Hilbert space by introducing the bilinear form

$$
\begin{aligned}
{\left[f^{\prime}, f\right] } & =\sum_{s}\left\langle f^{\prime}(s), g(s)\right\rangle=\sum_{u}\left\langle g^{\prime}(u), f(u)\right\rangle \\
& =\sum_{s, u}\left\langle g^{\prime}(u), V(s-u) g(s)\right\rangle .
\end{aligned}
$$

This form is unchanged if the same $f$ and $f^{\prime}$ are represented by different functions $g$ and $g^{\prime}$. It satisfies all the axioms for a scalar product on a Hilbert space, including positivity. In particular, $[f, f]=0$ if and only if the function $f(s)$ is zero for all $s$. The extension space $\mathscr{H}$ is the completion of the manifold $F$ in the norm induced by the above scalar product.

The space $\mathscr{H}_{U}$ is embedded in $\mathscr{H}$ by the sequence of linear maps:

$$
\begin{aligned}
\varphi \rightarrow g_{\varphi}(s) & =\varphi \delta_{0, s} \\
\rightarrow f_{\varphi}(s) & =V(-s) \varphi,
\end{aligned}
$$

where $\delta_{0, s}$ is a Kronecker $\delta$. The embedding is isometric: $\left[f_{\varphi}, f_{\varphi}\right]=\langle\varphi, \varphi\rangle$.

The projection of any element of $\mathscr{H}$ represented by a function $f(s)$ in $F$ onto $\mathscr{H}_{U}$ turns out to be $f(0)$, represented by

$$
\left[E_{U} f\right](s)=V(-s) f(0) .
$$

9 The relevant theorem is Theorem IV of [10]. Our summary follows $\S 6$ and 9 of that work. 
The unitary extension $U(t)$ is defined by

$$
\begin{aligned}
{[U(t) f](s)=f(s-t) } & =\sum_{u} V(t+u-s) g(u) \\
& =\sum_{u} V(u-s) g^{t}(u) ; \\
g^{t}(u) & \equiv g(u-t) .
\end{aligned}
$$

It is clear from the definition that $U(t)$ leaves the manifold $F$ invariant and that it is a representation of the translation group. It is not hard to show that it is unitary in the scalar product (3), and that it is weakly continuous if $V(t)$ is.

That $V(t)=E_{U} U(t) E_{U}$ follows from the calculation:

$$
\left[E_{U} U(t) E_{U} f\right](s)=V(-s)[V(t) f(0)] .
$$

For later use, we need the matrix element

$$
\left[f^{\prime}, U(t) f\right]=\sum_{i, j}\left\langle g_{j}^{\prime}, V\left(s_{i}+t-s_{j}^{\prime}\right) g_{i}\right\rangle,
$$

where we have introduced the notation

$$
g_{i}=g\left(s_{i}\right), g_{j}^{\prime}=g^{\prime}\left(s_{j}^{\prime}\right) \text { for the nonvanishing values of } g \text { and } g^{\prime} .
$$

Our proof of the theorem goes by two lemmas.

Lemma 1. There are nonvanishing vectors $\psi$ in $\mathscr{H}_{D} \equiv\left(I-E_{U}\right) \mathscr{H}$ which are localized in time in the sense that

$$
\langle\psi, U(t) \psi\rangle=0
$$

for $|t|$ large enough.

Proof. Consider those vectors in $\mathscr{H}$ represented by a finite sum

$$
f(s)=\sum_{i} V\left(-s+s_{i}\right) g_{i},
$$

where $g_{i} \in \mathscr{H}_{U}$ and where all $s_{i}$ have the same sign (zero is allowed). Let $F_{+}$be the linear submanifold of $F$ where all $s_{i} \geqq 0$; and let $F_{-}$be that where all $s_{i} \leqq 0$.

We claim that the matrix element of $U(t)$, projected onto $\mathscr{H}_{D}$, between any two vectors in $F_{+}$or between any two vectors in $F_{-}$,

$$
M(t) \equiv\left[f^{\prime},\left(I-E_{U}\right) U(t)\left(I-E_{U}\right) f\right],
$$

vanishes for $|t| \geqq \max \left[\sup _{i}\left|s_{i}\right|, \sup _{j}\left|s_{j}^{\prime}\right|\right]$.

Let's look at $F_{+}$; the argument is the same for $F_{-}$. Substituting the formula

$$
\left[E_{U} f\right](s)=V(-s) \sum_{i} V\left(s_{i}\right) g_{i}
$$


into (6), we get for $M(t)$ :

$$
\begin{aligned}
M(t)= & \sum_{i, j}\left\{\left\langle g_{j}^{\prime}, V\left(s_{i}+t-s_{j}^{\prime}\right) g_{i}\right\rangle\right. \\
& -\left\langle g_{j}^{\prime}, V\left(-s_{j}^{\prime}+t\right) V\left(s_{i}\right) g_{i}\right\rangle \\
& -\left\langle g_{j}^{\prime}, V\left(-s_{j}^{\prime}\right) V\left(s_{i}+t\right) g_{i}\right\rangle \\
& \left.+\left\langle g_{j}^{\prime}, V\left(-s_{j}^{\prime}\right) V(+t) V\left(s_{i}\right) g_{i}\right\rangle\right\} .
\end{aligned}
$$

We have used the fact that $V(s)^{*}=V(-s)$. Next, we look at a fixed value of $i$ and $j$ in the sum, and we distinguish two cases:

(i) Choose $-t \geqq s_{i} \geqq 0$, so we can use the semigroup property to write $V\left(s_{i}+t-s_{j}^{\prime}\right)=V\left(-s_{j}^{\prime}\right) V\left(s_{i}+t\right)$ and $V\left(-s_{j}^{\prime}+t\right)=V\left(-s_{j}^{\prime}\right) V(+t)$. The four terms add to zero by inspection.

(ii) Choose $t \geqq s_{j}^{\prime} \geqq 0$, so we can write $V\left(s_{i}+t-s_{j}^{\prime}\right)=V\left(-s_{j}^{\prime}+t\right) V\left(s_{i}\right)$ and $V\left(s_{i}+t\right)=V(+t) V\left(s_{i}\right)$. Again we get zero.

Clearly, the sum over $i$ and $j$ vanishes if we take $|t|$ not less than the largest of the $s_{i}$ and $s_{j}^{\prime}$.

All that remains is to verify that some of these vectors have a nonvanishing projection into $\mathscr{H}_{D}$. Consider the vector

$$
f(s)=V(-s) \varphi+V(-s+a) \varphi,
$$

with $\varphi \in \mathscr{H}_{U}$. It belongs to $F_{+}$or $F_{-}$, respectively, if $a$ is positive or negative. Then

$$
\left[E_{U} f\right](s)=V(-s)[I+V(a)] \varphi,
$$

and a short calculation gives

$$
\left[f,\left(I-E_{U}\right) f\right]=\left\langle\varphi,\left[I-V(a)^{*} V(a)\right] \varphi\right\rangle .
$$

Thus, if there is any positive number $a$ for which $V(a)$ is not unitary, either $V(a)^{*} V(a) \neq I$ or $V(-a)^{*} V(-a)=V(a) V(a)^{*} \neq I$; and there must be some vector $\varphi$ such that the matrix element above does not vanish. That proves Lemma 1, if we put $\psi=\left(I-E_{U}\right) f$.

Lemma 2. Let $U(t)=\exp (-i E t)$ be a weakly continuous, one parameter, unitary group on a Hilbert space $\mathscr{H}$. Let $\langle\psi, U(t) \psi\rangle=0$ for $|t| \geqq B\rangle 0$. Then either $\psi=0$ or the spectrum of $E$ is the whole real line.

Proof. This lemma is widely known among both physicists and mathematicians. It is a variant of the converse of the Paley-Wiener theorem. For completeness we give the proof.

Let $E=\int \lambda d E(\lambda)$ be the spectral decomposition of the self adjoint operator E. Then

$$
\langle\psi, U(t) \psi\rangle=\int e^{-i \lambda t} d\langle\psi, E(\lambda) \psi\rangle
$$


is the Fourier transform of a bounded measure. If it vanishes outside a bounded region in $t$, the measure is entire analytic. It either vanishes everywhere, in which case $\psi=0$, or its support is the whole line from plus to minus infinity, in which case all those points are in the continuous spectrum of $E$.

\section{Unitary Extension of Zwanziger's Representation}

We find the particular construction of Sz.-Nagy used in Section II a bit awkward for looking at specific examples, such as Zwanziger's representation. Although Sz.-Nagy has other versions of the construction that might be more suitable, we proceed instead by guessing the answer on "physical" grounds, and verifying that it is correct.

Thus, let $\mathscr{H}_{V}$ be the space of four-velocity wave functions corresponding to Zwanziger's nonunitary representation for complex mass $\Gamma=m-i \gamma$ and real spin $S$, as described in Section I. We are looking for an embedding of $\mathscr{H}_{U}$ into a larger space $\mathscr{H}$, and a minimal, unitary extension to $\mathscr{H}$ of the representation

$$
V(b, A)=T(b) U(A)
$$

of the Poincare group, where $A \in S L(2, C)$ corresponds to a homogeneous Lorentz transformation, represented by the unitary operator $U(A)$. The homogeneous transformations do not get extended, being already unitary; and to get a relativistic extension of the translations, we extend the semigroup of nonnegative, proper time evolution operators, by the prescription below.

We can write the action of the translations in the form

$$
[T(b) \varphi](u)=e^{i \Gamma \tau_{u}} \varphi(u),
$$

where $\tau_{u}=u \cdot b$, and we can emphasize the evolution in the center of momentum frame as follows. Let $\mathbb{C}$ be the one-dimensional Hilbert space of complex numbers with the usual scalar product. We can trivially write

$$
\mathscr{H}_{U}=\mathbb{C} \otimes \mathscr{H}_{U},
$$

and we define the action of the proper time evolution on $\mathbb{C}$ by

$$
V(\tau) z=e^{-i \Gamma \tau} z,
$$

for $z \in \mathbb{C}$. Suppose we have a minimal extension of the nonunitary semigroup $V(\tau)$ for $\tau \geqq 0$ from $\mathbb{C}$ to a space $\mathscr{H}_{\mathbf{M}} \supset \mathbb{C}$ and a unitary representation $\exp (-i \mathbb{M} \tau)$. Then we can define

$$
\mathscr{H}=\mathscr{H}_{\mathbf{M}} \otimes \mathscr{H}_{\mathrm{U}},
$$

23 Commun, math. Phys., Vol. 21 
and $U(b, A)=\exp (i \mathbb{M} u \cdot b) U(A)$, by which we mean the action

$$
U(b, A)[\varrho \otimes \varphi(\boldsymbol{u})]=[\exp i \mathbb{M} u \cdot b \varrho] \otimes[U(A) \varphi](\boldsymbol{u}),
$$

where $\varrho \in \mathscr{H}_{\mathbf{M}}$. This is clearly a unitary, continuous extension of $V(b, A)$, satisfying the multiplication law for the Poincaré group.

Because we expect an unstable particle to correspond to a particular mass distribution, we can guess that the extension space $\mathscr{H}_{\mathbf{M}}$ is naturally identified with $L_{2}\left(\mathbb{R}^{1}, d M\right)$, the elements of which are square integrable functions $\varrho(M)$ on $-\infty<M<\infty .{ }^{10}$ And we can guess that the unitary extension of $V(\tau)$ is

$$
[U(\tau) \varrho](M)=e^{-i M \tau} \varrho(M) .
$$

Now we have to find the one-dimensional subspace corresponding to $[V(\tau), \mathbb{C}]$, and check whether we indeed have a minimal extension.

Let the one-dimensional subspace to be identified with $\mathbb{C}$ be spanned by a fixed, unit vector $\varrho_{U} \in \mathscr{H}_{\mathbf{M}}$. The projection operator on this subspace is $\left|\varrho_{U} \times \varrho_{U}\right|$, and the equation $V(\tau)=\operatorname{pr} U(\tau)$ for $\tau \geqq 0$ is equivalent to

$$
\begin{aligned}
\left\langle\varrho_{U}, V(t) \varrho_{U}\right\rangle & =e^{-i \Gamma \tau}=\int_{-\infty}^{\infty} d M\left|\varrho_{U}(M)\right|^{2} e^{-i M \tau} \\
& \equiv F(\tau) .
\end{aligned}
$$

The right-hand side satisfies $F(-\tau)=\overline{F(\tau)}$, and thus $\left|\varrho_{U}\right|^{2}$ is the Fourier transform of

$$
F(\tau)=\theta(\tau) e^{-i \Gamma \tau}+\theta(-\tau) e^{-i \bar{\Gamma} \tau} .
$$

In other words,

$$
\left|\varrho_{U}\right|^{2}=\frac{\gamma}{\pi} \frac{1}{|M-\Gamma|^{2}},
$$

the familiar Lorentzian shape. These formulas were already written down by Matthews and Salam, from a less abstract viewpoint.

We can choose

$$
\varrho_{U}(M)=\sqrt{\frac{\gamma}{\pi}} \frac{1}{M-\Gamma},
$$

and the span of this vector fixes the subspace $\mathbb{C}$ in $\mathscr{H}_{\mathrm{M}}$. The extension $\left[U(\tau), \mathscr{H}_{\mathbf{M}}\right]$ of $[V(\tau), \mathbb{C}]$ defined in this way is minimal, because $\varrho_{U}$ does not vanish anywhere; and we can generate any measurable function by multiplying $Q_{U}$ with trigonometric polynomials formed from $e^{-i M \tau}$ and taking linear combinations (infinite sums, if necessary).

If we had made a different choice of phase for $\varrho_{U}$, multiplying by $\exp i \varphi(M)$, where $\varphi$ is any real, measurable function, we would have

\footnotetext{
to As usual, the choice of the measure $d M$ is free up to measure equivalence.
} 
induced a unitary transformation of $\mathscr{H}_{\mathrm{M}}$ by the map $\varrho \rightarrow \varrho \exp i \varphi$ and got a unitary-equivalent, minimal extension.

The extension space for $\left[V(b, A), \mathscr{H}_{U}\right]$, according to Eq. (1) and Eq. (11) is

$$
\mathscr{H}=L_{2}\left(\mathbb{R}^{4}, d M d^{3} u / 2 \sqrt{1+u^{2}}\right) \otimes \mathbb{C}^{2 S+1},
$$

and $\mathscr{H}_{U}$ is identified with the subspace of functions of the form $(M-\Gamma)^{-1} \varphi(\boldsymbol{u})_{\lambda}$. By the same reasoning as above, it is clear that the functions of the form $\exp (i M u \cdot b)(M-\Gamma)^{-1} \varphi(\boldsymbol{u})_{\lambda}$ span $\mathscr{H}$; and thus the extension $[U(b, A), \mathscr{H}]$ is also minimal.

Just as the theorem in Section II says, the spectrum of the mass operator $\mathbb{M}$ on $\mathscr{H}, \mathbb{M}$ being simply multiplication by $M$, contains all values from plus to minus infinity. The unitary representation $U(b, A)$ is a multiplicity-free, direct integral of irreducible representations of the Poincaré group for fixed spin $S$ and all masses $-\infty<M<\infty$. There is, of course, a doubling in the sense that the representations $[M, S]$ and $[-M, S]$ are antiunitary equivalent to each other.

At first glance, it is tempting to try to give a physical interpretation of the space $\mathscr{H}_{V}$, i.e., the wave functions in $\mathscr{H}$ having a Breit-Wigner mass distribution, by cutting off the mass below the threshold $M_{0}=\Sigma m_{j}$ of the $n$ decay products in whatever channel, and embedding the space $\mathscr{H}_{+}=\theta\left(M-M_{0}\right) \mathscr{H}$ in the angular momentum $S$ sector of the tensor product space of $n$ free particles. That is mathematically straightforward to do. If $n=2$, there is a finite multiplicity of ways (channels) to do that, while if $n \geqq 3$, the multiplicity of ways is countably infinite, according to the number of representations of the Poincare group having spin $S$ that appear in the decomposition of a tensor product.

Matthews and Salam proposed a view like that. Leaving aside the question whether the wave function ought to be cut off in a more sophisticated way, we could argue that this provides a concept of an elementary, unstable particle which in its ideal world has a distinguished mass distribution from plus to minus infinity, but which in the real world has open to it only decay channels with physical masses. We could say that the real world distorts our view of the elementary, unstable particle so that it does not obey an exponential decay law (which is close to the accepted view), but that its wave function has a "memory" of its ideal structure because it is analytic in the mass (up to a phase) and has a unique analytic continuation to negative masses giving the group theoretical distribution.

We find that interpretation uncomfortable, if the natural interpretation of the Sz.-Nagy extension from which we "derived" it, is not to be lost. If we say that the entire sector of the $n$-particle space in which the cutoff extension is embedded is the space of decay products, any con- 
nection between the physical time evolution and that of the ideal Sz.Nagy extension is obscured. If, on the other hand, we say that only that part of the $n$-particle channel orthogonal to the cut-off $\mathscr{H}_{U}$ is the space of decay products, as in the ideal Sz.-Nagy extension, we are still faced with the problem of specifying the physical time evolution. If it is to be simply that of the cut-off Sz.-Nagy extension, mathematically identified via the embedding with that of $n$ free particles, we have to reconcile two interpretations of the same space: one, that of the unstable particle with decay products; the other, that of $n$ free particles.

Perhaps such a line of thought can be sustained, but we prefer to follow the ideas of the Wigner-Weisskopf theory sketched in the Introduction.

\section{A Possible Model}

Let us discuss the possibility that the MSZ representation can be understood as describing the "interaction part" of the time evolution, so that the exponential decay law is realized in the approximation that all kinetic terms in the invariant Hamiltonian or mass operator are neglected. There is no reason in principle why the interaction should not have a spectrum from plus to minus infinity, so that objection to the MSZ representation could then be removed.

Thus, we reinterpret the variable $M$ in the wave functions of vectors in the unitary extension space to mean center of momentum interaction energy, and we change its name to $V$, replacing the notation $L_{2}\left(\mathbb{R}^{1}, d M\right)$ by $L_{2}\left(\mathbb{R}^{1}, d V\right)$, and reserving $M$ for the continuous spectrum of the free mass operator.

To specific the model, we apply the Wigner-Weisskopf theory, more or less in the form developed by Höhler [11], restricting ourselves always to the center of mass. Our Hilbert space has the same structure as before, $\mathscr{H}=\mathscr{H}_{U} \oplus \mathscr{H}_{D}$, and we have a realization in which the interaction is diagonal, $\mathscr{H}=L_{2}\left(\mathbb{R}^{1}, d V\right)$. In this realization, the one-dimensional space $\mathscr{H}_{V}$ is identified, as before, with the span of the Breit-Wigner wave function $\varrho_{V}(V)$.

Now we make a second identification of the components $\mathscr{H}_{U}$ and $\mathscr{H}_{D}$. The space $\mathscr{H}_{U}$ is interpreted as the only discrete eigenspace of the free mass operator, corresponding to the undecayed, unstable, elementary particle. We emphasize that by the identification $\mathscr{H}_{U}=\mathbb{C}$. The space $\mathscr{H}_{D}$ is interpreted as that of the free, center of mass wave functions of the decay products, belonging to the continuum of the free mass operator. We can realize it in a representation where the free mass is diagonal by the identification

$$
\mathscr{H}_{\boldsymbol{D}}=L_{2}\left[\mathbb{R}^{1}, \theta\left(M-M_{0}\right) d M\right] \equiv L_{2}\left(M_{0}, \infty\right), \quad M_{0}>0,
$$


where $M_{0}$ is the threshold of the free decay products. This gives a realization

$$
\mathscr{H}=\mathbb{C} \oplus L_{2}\left(M_{0}, \infty\right)
$$

in which the free mass operator $\mathbb{M}_{0}$ has the form

$$
\mathbb{M}_{0}=m E_{U} \oplus \mathbb{M}_{-0}^{D}, \quad m>M_{0},
$$

with the action

$$
\begin{aligned}
\mathbb{M}_{0} z & =m z, \quad z \in \mathbb{C} ; \\
\left(\mathbb{M}_{0} f\right)(M)=\left(\mathbb{M}_{0}^{D} f\right)(M) & =M f(M), \quad f \in L_{2}\left(M_{0}, \infty\right) .
\end{aligned}
$$

As we described in Section III, only the space $\mathscr{H}_{D}=L_{2}\left(M_{0}, \infty\right)$ is to be embedded in an angular momentum $S$ sector of a space of $n$ free particles.

Now we have two realizations of $\mathscr{H}$, corresponding to the diagonalization of the interaction and of the free mass, respectively. We determine the dynamics when we give the correspondence between the two representations. The mapping between the two representation of $\mathscr{H}_{U}$ is trivial, so we need only choose an isometry between the two representations of $\mathscr{H}_{D}$. All such isometries are parametrized by picking O.N. bases in each realization and putting them in one-to-one correspondence. Thus, let $\varphi_{i}, i=1,2, \ldots$, be on O.N. basis in $L_{2}\left(M_{0}, \infty\right)$; and let $\psi_{i}$ be an O.N. basis in the subspace of $L_{2}\left(\mathbb{R}^{1}, d V\right)$ orthogonal to $\varrho_{U}$. Let $U$ be the isometric map

defined by

$$
U:\left[\mathbb{C} \oplus L_{2}\left(M_{0}, \infty\right)\right] \rightarrow L_{2}\left(\mathbb{R}^{1}, d V\right)
$$

$$
\begin{aligned}
U z & =z \varrho_{U}, \quad z \in \mathbb{C}, \\
U \varphi_{i} & =\psi_{i} .
\end{aligned}
$$

Then on $\mathscr{H}=\mathbb{C} \oplus L_{2}\left(M_{0}, \infty\right)$, we can write the total mass in the form

$$
\mathbb{M}=\mathbb{M}_{0}+U^{-1} V U \equiv \mathbb{M}_{0}+\mathbb{V} .
$$

The freedom in the interaction operator $\mathbb{V}$ is exactly the freedom of unitary transformations that commute with the projection operator $E_{U}$ for $\mathscr{H}_{U}$.

To specify the model further, and hopefully make it a physically reasonable description of an unstable particle, we have to look for a class of $U$ 's that guarantees several things:

(i) $\mathbb{M}$ ought to be defined as a positive, self-adjoint operator.

(ii) The wave operators should exist, and the asymptotic state corresponding to the initially undecayed state $\varrho_{V}$,

$$
\varrho_{U}^{\text {out }}=\lim _{\tau \rightarrow \infty} e^{i \mathbf{M}_{0} \tau} e^{-i \mathbf{M} \tau} \varrho_{U}=U(\infty, 0) \varrho_{U},
$$


ought to lie entirely in $\mathscr{H}_{D}$. The asymptotic states of vectors in $\mathscr{H}_{D}$ should remain in $\mathscr{H}_{D}$. In a theory with time inversion invariance, the same should be true of the incoming states. The wave operators $U(\infty, 0)$ and $U(-\infty, 0)$ should be partial isometries from $\mathscr{H}$ onto $\mathscr{H}_{D}$, so that the $S$ matrix

$$
S=U(\infty, 0) U(0,-\infty)=E_{D} S E_{D}
$$

is unitary on $\mathscr{H}_{D}$.

(iii) The positive time decay amplitude $\left\langle\varrho_{U}, e^{-i \mathbf{M} t} \varrho_{U}\right\rangle$ ought to be approximately exponential.

(iv) The appropriate, retarded Green's function ought to have an unstable particle pole on the second sheet. This property is well-known to be related to the approximate validity of the exponential decay law.

The last two requirements, (iii) and (iv), are not matters of principle, but rather features that experience indicates to be desirable.

Unfortunately, we have not been able to show as yet whether the well-defined mathematical problem posed by these conditions, especially positivity and self-adjointhess, has any solutions. If it does, then we clearly have a Wigner-Weisskopf theory with a particular class of interactions suggested, although indirectly, by the representation theory of the Poincaré group.

Even if self-adjointness and positivity can be solved, the rest of the program is complicated by the fact that $\mathbb{V}$ has nonvanishing matrix elements within $\mathscr{H}_{D}$, and not just between $\mathscr{H}_{U}$ and $\mathscr{H}_{D}$ and within $\mathscr{H}_{U}$. The exact solution of the weak-coupling approximation, where the matrix elements of the interaction within $\mathscr{H}_{D}$ are neglected, is well-known, whenever it is well-defined, from either the Wigner-Weisskopf or the Lee model [11, 12, 14-17]. In fact, we are going to conclude from the remark just below that the weak-coupling approximation is not mathematically defined in our case.

That fact is related to the basic question: how can $\mathbb{V}$ be chosen to make $\mathbb{I}$ self-adjoint? Although it is not the only possibility, it is natural to wonder whether $\mathbb{M}$ might be made self-adjoint by virtue of the Kato condition. Kato showed [18] that the sum of two self-adjoint operators $A+B$ is in turn self-adjoint if $A$ is bounded relative to $B$ (or vice versa); i.e., if the domain of $B$ is contained in the domain of $A$ and if

$$
\|A \varphi\| \leqq a\|\varphi\|+b\|B \varphi\|
$$

whenever $\varphi$ is in the domain of $A$, for some fixed $a$ and $b$ with $0 \leqq b<1$.

It is straightforward to see that whatever be the unitary transformation $U$ in the definition of $\mathbb{M}$, it is impossible to have $\mathbb{V}$ bounded relative to $\mathbb{M}_{0}$, because the domain of $\mathbb{I M}_{0}$ is the direct sum of $\mathscr{H}_{U}$ and the domain of $\mathbb{M}_{0}^{D}$ in $\mathscr{H}_{D}$ :

$$
\mathscr{D}\left(\mathbb{M}_{0}\right)=\mathscr{H}_{U} \oplus \mathscr{D}\left(\mathbb{M}_{0}^{D}\right),
$$


whereas the subspace $\mathscr{H}_{V}$ is not in the domain of $\mathbb{V}$, because

is not square integrable.

$$
\left[\mathbb{V}_{\varrho_{v}}\right](V)=\sqrt{\frac{\gamma}{\pi}} \frac{V}{V-\Gamma}
$$

On the other hand, we have not been able to settle the possibility that $\mathbb{V}$ could be chosen to have its domain inside that of $\mathbb{M}_{0}$, and to bound $\mathbb{M}_{0}$ in the Kato sense. We would call such a theory a strong coupling theory. We think that such a possibility has a certain appeal as a quasikinematic description for elementary unstable particles, for not only would the unstable particle representation of the Poincaré group play a role, it would dominate.

In any case, however $\mathbb{M}$ might be defined, we can see from this discussion that the weak coupling approximation is not defined as an operator theory in our perhaps nonexistent model. It corresponds to the decomposition

$$
\mathbb{V}=E_{U} \mathbb{V} E_{U}+E_{U} \mathbb{V} E_{D}+E_{D} \mathbb{V} E_{U}+E_{D} \mathbb{V} E_{D},
$$

with the last term thrown out. What is left cannot be a self-adjoint operator, because the fact that $\varrho_{U}$ is not in $\mathscr{D}(\mathbb{V})$ means that $E_{D} \mathbb{V} E_{U}$ is not an operator, although its matrix elements between $\mathscr{H}_{D}$ and $\mathscr{H}_{U}$ do exist for the dense manifold of wave functions in $\mathscr{H}_{D}$ having sufficiently rapid decrease at infinity in the $V$ representation. Another way to see that is to verify that while $E_{U} \mathbb{V} E_{D}$ is defined as an unbounded operator on the dense domain just mentioned, its adjoint is zero, according to the mathematical definition.

\section{Appendix:}

Trouble with an Exponential Decay Law

The theorem proved here relaxes the condition that the evolution of the unstable state obey a semigroup law, but imposes a more detailed law of decay. It is well-known [9], and we just give a modern proof for completeness.

Theorem. Let $V(t)=E_{U} U(t) E_{U}$, where $U(t)$ is a strongly continuous, one parameter, unitary group on a Hilbert space $\mathscr{H} \supset \mathscr{H}_{\mathrm{U}}$.

(i) If $\|\boldsymbol{V}(t) \varphi\| \leqq C \exp (-\gamma t)$ for some $\gamma>0$ and all $t>0$, and for some nonvanishing $\varphi$ in $\mathscr{H}_{U}$, then the spectrum of the infinitesimal generator of $U(t)$ is the whole real line.

(ii) The same conclusion holds if we have exponential decrease only in the weak sense that there are two vectors $\psi$ and $\varphi$ in $\mathscr{H}_{U}$ with $\langle\psi, \varphi\rangle \neq 0$ and $|\langle\psi, V(t) \varphi\rangle| \leqq C \exp (-\gamma t)$ for some $\gamma>0$ and all $t>0$. 
Proof. We can reduce the first case to the second. In the first case, if we insert a complete set of vectors in $\mathscr{H}_{U}$ we get

$$
\|V(t) \varphi\|^{2}=\sum_{i}\left\langle\varphi, V(t)^{*} \psi_{i}\right\rangle\left\langle\psi_{i}, V(t) \varphi\right\rangle .
$$

Each term in the sum is nonnegative, and hence has at least as good an exponential bound as the sum. If $\varphi \neq 0$, there must be some value of the index $i$ for which $\left\langle\psi_{i}, \varphi\right\rangle \neq 0$. Put $\psi_{i}=\psi$, and we are in case (ii).

Now $M(t) \equiv\langle\psi, V(t) \varphi\rangle=\langle\psi, U(t) \varphi\rangle$, because $\varphi$ and $\psi$ are in $\mathscr{H}_{U}$. Let

$$
M(t)=\int d \lambda \mathscr{M}(\lambda) e^{-i \lambda t},
$$

where $\mathscr{M}(\lambda)$ represents the bounded measure associated with the spectral decomposition of the generator $E$ :

$$
d\langle\psi, E(\lambda) \varphi\rangle=d \lambda \mathscr{M}(\lambda) .
$$

Since the matrix element $M(t)$ is a uniformly bounded, continuous function, the decomposition

$$
M(t)=[\theta(t)+\theta(-t)] M(t)
$$

is well defined in the sense of tempered distributions; and if we set

$$
\tilde{M}_{t}(\lambda)=\frac{1}{2 \pi} \int_{-\infty}^{\infty} d t \theta( \pm t) M(t) e^{i \lambda t},
$$

we get

$$
\mathscr{M}(\lambda)=\tilde{M}_{+}(\lambda)+\tilde{M}_{-}(\lambda) .
$$

It is clear from the definition that for real $\lambda$ the tempered distribution $\tilde{M}_{+}(\lambda)$ is the boundary value of a function analytic in the upper half plane, while $\tilde{M}_{-}(\lambda)$ is the boundary value of an analytic function in the lower half plane.

Suppose some nonempty, open set $\mathcal{O}$ of real values $\lambda$ is not in the spectrum of $E$. Then $\mathcal{O}$ is excluded from the support of $\mathscr{M}(\lambda)$, and

$$
\tilde{M}_{+}(\lambda)=-\tilde{M}_{-}(\lambda)
$$

for $\lambda \in \mathcal{O}$. It follows from the edge-of-the-wedge theorem for distribution boundary values [19] that $\tilde{M}_{+}$and $-\tilde{M}_{-}$are different pieces of one function analytic in the whole $\lambda$ plane, except possibly for that part of the real axis in the spectrum of $E$; and we see that $\mathscr{M}(\lambda)$ is just the discontinuity of this analytic function, as is well known.

So far, we haven't used anything except the fact that $U(t)$ is unitary and continuous. If we have a decreasing exponential bound on $M(t)$ for positive $t$, it follows that $\widetilde{M}_{+}(\lambda)$ is analytic in the half plane $\operatorname{Im} \lambda>-\gamma$, which includes the real axis; and hence the function we just defined via 
analytic continuation and the edge-of-the-wedge theorem is an entire function. We conclude that any open set $\mathcal{O}$ not contained in the spectrum of $E$ must be empty, for otherwise the discontinuity is zero and $\mathscr{M}(\lambda)$ is zero, contradicting our hypothesis that $\langle\psi, \varphi\rangle=\int d \lambda \mathscr{M}(\lambda)$ is nonzero.

Hence we find that the spectrum of $E$ is the whole line.

\section{References}

1. Matthews, P. T., Salam, A.: Phys. Rev. 112, 283 (1958); 115, 1079 (1959).

2. Zwanziger, D.: Phys. Rev. 131, 2818 (1963).

3. Beltrametti, E., G., Luzzato, G.: Nuovo Cimento 36, 1217 (1965).

4. Kawai, T., Gotō, M.: Nuovo Cimento 60 B, 21 (1969).

5. Lurçat, F.: Phys. Rev. 173, 1461 (1968).

6. Chew, G.F.: The analytic $S$ matrix. Chap. 1. New York: W. A. Benjamin, Inc. 1966.

7. Newton, R.G.: Scattering theory of waves and particles. New York: McGraw-Hill Book Company 1966.

8. Beltrametti, E. G.: Nuovo Cimento 25, 1393 (1962).

9. Lévy, M.: Nuovo Cimento 14, 612 (1960).

10. Sz.-Nagy, B.: Linear transformations in Hilbert space which extend beyond this space. Appendix to Riesz,F. and Sz.-Nagy, B., Functional analysis. New York: Frederick Ungar Publishing Co. 1960.

11. Höhler, G.: Z. Physik 152, 542 (1958).

12. Messiah, A.: Quantum mechanics. Vol. II, Chap. XXI, Sect. 13. Amsterdam: NorthHolland Publishing Company 1962.

13. Coester, F: Helv. Phys. Acta 38, 7 (1965).

14. Glaser, V., Källén, G.: Nucl. Phys. 2, 706 (1956).

15. Araki, H., Munakata, Y., Kawaguchi, M., Gotō,T.: Progr. Theoret. Phys. (Kyoto) 17, 419 (1957).

16. Heitler, W.: Quantum theory of radiation (Oxford, 1964, 2nd edition).

17. Lévy, M.: Nuovo Cimento 13, 115 (1959).

18. Kato, T.: Perturbation theory for linear operators. Chap. V, $\S 4.1$. New York: Springer-Verlag 1966.

19. Streater, R.F., Wightman, A. S.: PCT, spin and statistics and all that, p. 82. New York: W. A. Benjamin 1964.

David N. Williams

The University of Michigan

Dept. of Physics

Physics-Astronomy Building

Ann Arbor, Mich. 48104, USA 